Transactions

Elsevier Editorial system(tm) for ISA

Manuscript Draft

Manuscript Number: ISATRANS-D-16-01021R3

Title: Fractional order Implementation of Integral Resonant Control - A Nanopositioning Application

Article Type: SI: Fractional Order SSCTA

Section/Category: Application

Keywords: fractional order control; smart structures; piezoelectric actuators; robust control.

Corresponding Author: Mr. Andres San-Millan, M.D.

Corresponding Author's Institution: Universidad de Castilla-La Mancha

First Author: Andres San-Millan, M.D.

Order of Authors: Andres San-Millan, M.D.; Vicente Feliu-Batlle; Sumeet S Aphale

Abstract: By exploiting the co-located sensor-actuator arrangement in typical flexure-based piezoelectric stack actuated nanopositioners, the pole-zero interlacing exhibited by their axial frequency response can be transformed to a zero-pole interlacing by adding a constant feed-through term. The Integral Resonant Control (IRC) utilizes this unique property to add substantial damping to the dominant resonant mode by the use of a simple integrator implemented in closed loop. IRC used in conjunction with an integral tracking scheme, effectively reduces positioning errors introduced by modelling inaccuracies or parameter uncertainties. Over the past few years, successful application of the IRC control technique to nanopositioning systems has demonstrated performance robustness, easy tunability and versatility. The main drawback has been the relatively small positioning bandwidth achievable. This paper proposes a fractional order implementation of the classical integral tracking scheme employed in tandem with the IRC scheme to deliver damping and tracking. The fractional order integrator introduces an additional design parameter which allows desired pole-placement, resulting in superior closed loop bandwidth. Simulations and experimental results are presented to validate the theory. A 250\% improvement in the achievable positioning bandwidth is observed with proposed fractional order scheme.

Suggested Reviewers: 


\section{Cover Letter}

\section{Fractional order Implementation of Integral Resonant Control - A Nanopositioning Application}

\section{Andres San-Millan}

Instituto de Investigaciones Energeticas y Aplicaciones Industriales (INEI), Campus Universitario de Ciudad Real, 13071 Ciudad Real, Spain

andres.sanmillan.rodriguez@gmail.com

+34 926295300 (ext. 6341)

\section{Vicente Feliu-Batlle}

Escuela Tecnica Superior de Ingenieros Industriales, Universidad de Castilla-La

Mancha, Ciudad Real, 13071 Spain

vicente.feliuduclm.es

+34 926295300 (ext. 3870)

\section{Sumeet S. Aphale}

Center for Applied Dynamics Research, School of Engineering, University of Aberdeen, Aberdeen, AB24 3UE, U.K.

s.aphale@abdn.ac.uk

$+44(0) 1224274479$

\section{Abstract}

By exploiting the co-located sensor-actuator arrangement in typical flexure-based piezoelectric stack actuated nanopositioners, the pole-zero interlacing exhibited by their axial frequency response can be transformed to a zero-pole interlacing by adding a constant feed-through term. The Integral Resonant Control (IRC) utilizes this unique property to add substantial damping to the dominant resonant mode by the use of a simple integrator implemented in closed loop. IRC used in conjunction with an integral tracking scheme, effectively reduces positioning errors introduced by modelling inaccuracies or parameter uncertainties. Over the past few years, successful application of the IRC control technique to nanopositioning systems has demonstrated performance robustness, easy tunability and versatility. The main drawback has been the relatively small positioning bandwidth achievable. This paper proposes a fractional order implementation of the classical integral tracking scheme employed in tandem with the IRC scheme to deliver damping and tracking. The fractional order integrator introduces an additional design parameter which allows desired pole-placement, resulting in superior closed loop bandwidth. Simulations and experimental results are presented to validate the theory. A $250 \$ 1 \% \$$ improvement in the achievable positioning bandwidth is observed with proposed fractional order scheme.

\section{Acknowledgements:}

This paper was sponsored by the Spanish FPU12/00984 Program (Ministerio de Educacion, Cultura y Deporte). It was also sponsored by the Spanish Government Research Program with the Project DPI2012-37062-CO2-01 (Ministerio de Economia y Competitividad) and by the European Social Fund. 


\section{Title Page}

\section{Fractional order Implementation of Integral Resonant Control - A Nanopositioning Application}

Andres San-Millan (Corresponding author)

Instituto de Investigaciones Energeticas y Aplicaciones Industriales (INEI), Campus Universitario de Ciudad Real, 13071 Ciudad Real, Spain

andres.sanmillan.rodriguezegmail.com

+34926295300 (ext. 6341)

Vicente Feliu-Batlle

Escuela Tecnica Superior de Ingenieros Industriales, Universidad de Castilla-La Mancha, Ciudad Real, 13071 Spain

vicente.feliuduclm.es

+34 926295300 (ext. 3870)

Sumeet S. Aphale

Center for Applied Dynamics Research, School of Engineering, University of Aberdeen, Aberdeen, AB24 3UE, U.K.

s.aphaledabdn.ac.uk $+44(0) 1224274479$ 


\section{Highlights}

- New results on the application of fractional-order controllers to nanopositioners

- Design of a fractional-order IRC control scheme and application to a nanopositioner

- Expansion of the available bandwidth beyond the resonant frequency of the plant

- Experimental validation and robustness analysis of the proposed controller 


\title{
Fractional order Implementation of Integral Resonant Control - A Nanopositioning Application
}

\begin{abstract}
By exploiting the co-located sensor-actuator arrangement in typical flexure-based piezoelectric stack actuated nanopositioners, the pole-zero interlacing exhibited by their axial frequency response can be transformed to a zeropole interlacing by adding a constant feed-through term. The Integral Resonant Control (IRC) utilizes this unique property to add substantial damping to the dominant resonant mode by the use of a simple integrator implemented in closed loop. IRC used in conjunction with an integral tracking scheme, effectively reduces positioning errors introduced by modelling inaccuracies or parameter uncertainties. Over the past few years, successful application of the IRC control technique to nanopositioning systems has demonstrated performance robustness, easy tunability and versatility. The main drawback has been the relatively small positioning bandwidth achievable. This paper proposes a fractional order implementation of the classical integral tracking scheme employed in tandem with the IRC scheme to deliver damping and tracking. The fractional order integrator introduces an additional design parameter which allows desired pole-placement, resulting in superior closed loop bandwidth. Simulations and experimental results are presented to validate the theory. A $250 \%$ improvement in the achievable positioning bandwidth is observed with proposed fractional order scheme.
\end{abstract}

Keywords: fractional order control; smart structures; piezoelectric actuators; strain gauges; robust control.

\section{Introduction}

Control strategies applicable to nanopositioners need to deliver high positioning bandwidth and low-error positioning performance and be robust in the presence of parameter uncertainties. The high positioning bandwidth is a requirement stemming from the popular use of a wide-band actuation signal, the triangle waveform. The triangle wave trajectory in one axis of the nanopositioner is combined with a ramp-like trajectory in the other axis to generate an $x-y$ raster scan, the most common scanning pattern in Atomic Force Microscopy, [1]. Positioning errors in such scans mainly arise from three inherent sources viz: (i) Lightly-damped first resonant mode of each individual axis that dominates the axis dynamics within the bandwidth of interest, (ii) Intrinsic nonlinearities such as hysteresis and creep in the employed piezoelectric actuator and (iii) Cross-coupling between the $x$ and $y$ axis of the nanopositioner, [2]. The cross-coupling effects can be minimized during the mechanical design stage by utilizing a low-cross-coupling $(\approx-40 d B)$ design between axes, virtually deeming them decoupled, [3].The detrimental effects of the resonant mode as well as the nonlinearities are usually minimized by implementing a suitable control scheme. The typical approach is to implement a dual-loop control strategy. In this approach, the inner loop imparts adequate damping to the lightly-damped resonant mode through any one of a number of damping controllers proposed till date; such as Positive Position Feedback (PPF), Positive Velocity and Position Feedback (PVPF), Integral Resonant Control (IRC), Integral Force Feedback (IFF) etc, [4-7]. The outer loop is employed to minimize positioning error due to the nonlinearities by incorporating a tracking controller, typically a high-gain integrator, [8].

Over the past few years, IRC has emerged as a low-order, guaranteed stable, easily adjustable, highperformance damping controller with robustness in the presence of plant parameter uncertainties. Consequently, the control strategy in which the IRC is combined with a suitably gained integral tracking controller has gained popularity in nanopositioning applications, $[8,9]$. To date, the relationship between its parameters, namely the feed-through term and the gains of the damping and tracking controllers, and the achievable performance of the system has been analytically derived [10]. The sequential design of individual damping (IRC) and tracking (integrator) control loops was

July 21, 2017 
shown to deliver sub-optimal positioning performance and thus, a simultaneous design of the damping and tracking controllers was recently proposed, [11]. Furthermore, it was recently shown that in order to achieve an ideal flat band response, the closed loop poles of the damped and tracked nanopositioner axis could be placed in a Butterworth pattern, i.e., all the closed loop poles are equally spaced on a circle, with radius equal to the natural frequency of the Butterworth filter, $[12,13]$.

The main disadvantage of the IRC is indeed produced by its inherent simplicity. Because the IRC presents only three parameters to tune while needing four closed loop poles to be placed, all the poles cannot be placed arbitrarily. This leads to a very small closed-loop positioning bandwidth, usually close to the half of the resonant frequency of the uncontrolled open loop system, [13]. The presence of a pure-time delay in the system that creeps in due to the hardware utilized in the actual control implementation modifies the position of the closed loop poles, further limiting the achievable bandwidth, [14]. Yet, due to the many advantages and simplicity of the IRC scheme, it is desirable to find a way to expand the achievable bandwidth of this control scheme and at the same time keep the in-bandwidth closed-loop response as flat as possible. Fractional order controllers are usually utilized to design very robust schemes by designing the phase margin of the closed loop system $[15,16]$ and have been widely employed to control smart structures where the vibrations are a major issue, [17-19]. Using this as a motivation, this paper proposes a modified control scheme that replaces the standard integral tracking controller with a fractional order integrator.

This modification facilitates an additional design parameter that has the potential of increasing the achievable positioning bandwidth. Additionally, design rules to compensate the effects of hardware-induced time delay are also formulated, resulting in increase of the positioning bandwidth achieved.

This paper is organized as follows. Section 2 presents the mathematical model for one axis of a piezo-actuated nanopositioner. The new control scheme (abbreviated to FIRC) that combines the traditional IRC damping scheme with a fractional order integral tracking controller is formulated in Section 3. Section 4 describes the experimental setup used to validate the theory developed in this work. The simulated results produced by the proposed controller are presented in Section 5, and the experimental results and conclusions are presented in Sections 6 and 7.

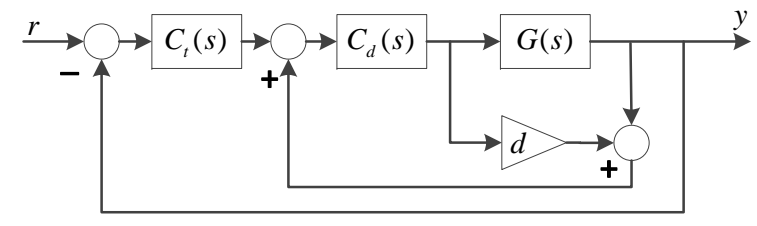

Figure 1: Block diagram for the traditional IRC damping controller in addition to integral tracking controller scheme.

\section{Problem statement}

The frequency response of one axis of a typical flexure-based piezo-stack actuated nanopositioner can be modelled as an infinite sum of second order terms with lightly damped resonant modes:

$$
G_{M}(s)=e^{-\tau s} \sum_{h=1}^{M} \frac{\sigma_{h}^{2}}{s^{2}+2 \zeta_{h} \omega_{h} s+\omega_{h}^{2}}
$$

where $M$ denotes the number of resonant modes considered in the model (ideally $M \rightarrow \infty$ ), $\sigma_{h}^{2}$ corresponds to the gain of each resonant mode, $\zeta_{h}$ is the damping ratio of each mode, $\omega_{h}$ is the natural frequency of each resonant mode, and $\tau$ is the value of time delay in the system. Controlling this type of system is a challenging task due to the infinite number of resonant poles of the system combined with the infinite number of poles introduced by the delay which is a transcendental transfer function.

The IRC is a pure damping-only control scheme that uses an adequate feed-through term to reverse the polezero pattern exhibited by co-located systems (systems with co-located sensors and actuators) into a zero-pole pattern. A suitably gained integrator, $C_{d}(s)$, implemented in positive feedback with this modified zeropole patterned system is capable of adding significant damping to multiple resonant modes of the system, [20]. To this damped system, a high-gain integrator, $C_{t}(s)$, is implemented to impart input command tracking forcing the system output (position in the case of nanopositioners) to follow the system input (desired trajectory), $[8,9,21]$. The combined damping and tracking control scheme is shown in Fig. 1.

As mentioned earlier in Section 1, even if a simplified model of (1) is considered (a single resonant mode with no delay), the number of closed-loop poles is greater than the number of parameters that can be tuned (four poles and three parameters), leading to a relatively small achievable closed-loop positioning bandwidth. In practice, though the simplified model considering only the first dominant resonant mode as given in 1 is accept- 
able (due to the substantial spacing between consecutive resonant modes), the effect of the delay cannot be neglected, deeming the methodologies proposed in [10] and [13] inapplicable. These drawbacks are overcome using the fractional order IRC design proposed in the following section.

\section{Fractional IRC Design}

As stated in Section 1, two different trajectory commands namely the triangular wave and staircase are used to generate a raster scan. It is straightforward to conclude that the design parameters of the control scheme should be tuned depending on the type of trajectory to be followed. In almost all control schemes targeted towards nanopositioning, the controller parameters are optimized to track only triangular trajectories and therefore, the main focus is on maximizing the achievable positioning bandwidth. The experimental and simulated results in this work demonstrate that this introduces a trade-off between the performance in the frequency domain (utilized to evaluate triangular signals) and in the time domain (utilized to evaluate step signals). This trade-off between parameters needed to track each of the two signals signals perfectly, directly impacts the design of the control scheme.

\subsection{Step 1: Maximize the achievable bandwidth}

As the traditional IRC controller is usually designed considering only the first resonant mode of the system, $[10,13]$, the proposed fractional order IRC is also designed considering the particular case where $M=1$ in (1); which leads to:

$$
G(s)=e^{-\tau s} \frac{\sigma^{2}}{s^{2}+2 \zeta \omega s+\omega^{2}}
$$

where the index $h=1$ has been omitted for the sake of clarity. Considering the system (2) and the general implementation of the IRC scheme, the closed loop block diagram with all the control parameters is shown in Fig. 2 , where $K_{t c}$ is the gain of the tracking controller, $\alpha$ is the fractional order of the tracking controller, $K_{d c}$ is the gain of the damping controller, $\beta$ is the fractional order of the damping controller, and $d$ is the feed-through term. In this case, if $\alpha=1$ and $\beta=1$, the traditional integer order IRC scheme is obtained, and by using $0<\alpha<1$ and $0<\beta<1$, different fractional order implementations of the IRC scheme are obtained. Since the outer tracking loop governs the overall closed loop tracking bandwidth, [11], this work focuses on the impact of converting the first order tracking integrator implemented in the outer loop to a fractional order one ( $\beta=1$ and $0<\alpha<1$ ). To further simplify the notations, $\beta$ is omitted from the analysis henceforth. The closed loop transfer function of the system can then be written as:

$$
\begin{array}{r}
H(s)=\frac{K_{d c} K_{t c} \sigma^{2}}{e^{\tau s} s^{\alpha}\left(s^{3}+s^{2}\left(2 \zeta \omega-d K_{d c}\right)+s\left(\omega^{2}-2 \zeta \omega d K_{d c}\right)\right.} \\
\left.-d K_{d c} \omega^{2}\right)+K_{d c} \sigma^{2}\left(K_{t c}-s^{\alpha}\right)
\end{array}
$$

It can be seen that the case where $\alpha=1$ leads to the traditional IRC scheme where the maximally flat band response can be achieved by arranging the closed loop poles in a Butterworth pattern (equally spaced on a circle of radius $\left.\omega_{B}\right)$. However, for values of $0<\alpha<1$ even if the delay is neglected $(\tau=0)$, the denominator of (3) takes the following form:

$$
\begin{array}{r}
H(s)_{d e n}=s^{3+\alpha}+s^{2+\alpha}\left(2 \zeta \omega-d K_{d c}\right)+ \\
s^{1+\alpha}\left(\omega^{2}-2 \zeta \omega d K_{d c}\right)-s^{\alpha} K_{d c}\left(d \omega^{2}+\sigma^{2}\right)+K_{d c} K_{t c} \sigma^{2}
\end{array}
$$

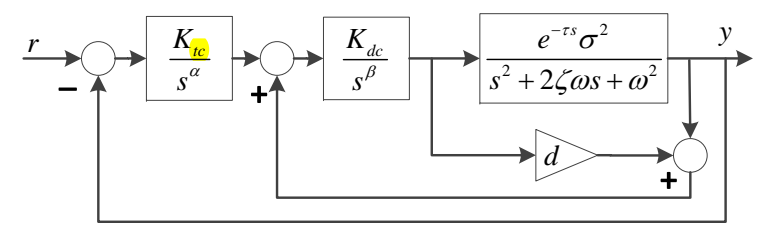

Figure 2: Block diagram of the FIRC scheme.

It was shown in [22], that the concept of analog Butterworth filter and its maximally flat-band response can be extended to fractional order systems, but the poles of these fractional order Butterworth filters need to be placed in very specific locations along $Q$ Riemann sheets (where $Q$ is related to the number of decimals of the fractional order of the filter). This imposes specific restrictive conditions on the structure of the denominator of fractional order Butterworth filters that cannot be satisfied by (4). It is important to note that if this condition is not satisfied in the simplest case without time delay, the case with an arbitrary time delay $\tau \neq 0$ will prove impossible.

However, if restriction of maximally flat-band response is relaxed, a controller can be designed such that the closed loop response achieves a compromise between flat in-bandwidth response and maximum achievable bandwidth. In order to do so, the parameters of the controller, i.e. $K_{t c}, \alpha, K_{d c}$ and $d$, are designed by optimizing the closed loop frequency response of (3). It is 
important to note that the system delay was included in the optimization procedure.

The idea of designing a filter by means of optimization has been successfully applied to fractional order filters in the past, aimed at reproducing integer order filter behaviour, $[23,24]$. Since the goal in this stage is to increase the achievable bandwidth and keeping a flat-band response, the approach utilized in the design of the parameters of the FIRC scheme is different, and is based on optimizing the maximum bandwidth, $\omega_{b w}$, which is defined as the lowest frequency at which the $-1 \mathrm{~dB}$ line is crossed by the magnitude response of the closed loop system, with two restrictions:

- The maximum allowed amplitude of the band pass ripple, $\delta_{\max }$ which is defined as the maximum value reached by the magnitude response of the closed loop system for frequencies below $\omega_{b w}$.

- The closed loop system must be stable.

It is important to note that the $\pm 3 \mathrm{~dB}$ bandwidth is widely employed to quantify the performance of different control schemes. Because of practical considerations (high accuracy and low error constraints typical of nanopositioning applications), the optimization procedure utilises the $\pm 1 \mathrm{~dB}$ band as an admissibility criterion. This is achieved by setting the restriction $\delta_{\max }=1 \mathrm{~dB}$, and by considering the $-1 \mathrm{~dB}$ line in the definition of $\omega_{b w}$. In other words, the magnitude response of the closed loop damped and tracked system is kept within $\pm 1 \mathrm{~dB}$ for as wide a range of frequencies as possible.

Once the goals and restrictions of the problem are set, different values of $\alpha$ within the range $[0,1]$ are chosen and the remaining parameters of the control scheme: $K_{t c}, K_{d c}$ and $d$ are computed so that the value of $\omega_{b w}$ is maximized under the restrictions that $\delta_{\max }=1$ and that the closed loop system is stable.

In order to illustrate the relationship between the fractional order of the FIRC and the bandwidth increase produced by it, the remaining parameters of the controller, i.e. $K_{t c}, K_{d c}$ and $d$, were obtained for different values of $\alpha$ as follows:

1. The parameters of the FIRC scheme, $K_{t c}, K_{d c}$, and $d$ were computed by placing the closed loop poles of (3) in a low-pass Butterworth pattern with the analytical expression that can be found in the Appendix A. This maximizes $\omega_{b w}$ considering a $\delta_{\text {max }} \approx 0 \mathrm{~dB}$.

2. Then, for the case $\alpha=1$, the $\pm 1 \mathrm{~dB}$ criterion is enforced, and the values of $K_{t c}, K_{d c}$, and $d$ obtained in the previous step are utilized as initial values in the maximization of $\omega_{b w}$ with the restriction of $\delta_{\max }=1 \mathrm{~dB}$ and ensuring system stability.

3. Successive decrements of $\Delta \alpha=0.01$ are applied to $\alpha$, and the values of $K_{t c}, K_{d c}$, and $d$ obtained in the previous step are utilized as initial values in the maximization of $\omega_{b w}$ by using the gradient method under the restriction $\delta_{\max }=1 \mathrm{~dB}$.

4. The previous steps are repeated until $\alpha=0.4$ was reached.

This optimization algorithm was employed due to its simplicity and effectiveness in achieving the optimal solution for each desired value of $\alpha$. Limitations of the proposed optimization method are:

- Because of the small number of controller design parameters, only the closed loop poles of the first resonant mode of the nanopositioner can be placed arbitrarily, which justifies choosing $M=1$ for the optimization procedure. It was additionally verified that if all the identified modes of vibration of the system $(M=4)$ were considered in the optimization process, the obtained results present a difference less than $0.1 \%$ when compared with the results obtained considering $M=1$, but the computational burden of the optimization process is increased.

- The fractional order term in the system deems determining the position of all the closed loop poles computationally expensive. It is therefore advisable to utilize the phase response of the system in order to distinguish between stable and unstable closed loop systems.

Additionally, it is important to note that the fractional order integrator could be utilized in both the external and the internal loop. However, using an additional fractional exponent would add an extra degree of freedom to the design of the controller that would exponentially increase the computational burden of the optimization process. But as the outer loop governs the total bandwidth of the system, [10], employing the fractional order integrator in the outer loop to gain the extra degree of freedom that facilitates the management of achievable bandwidth proves to be a logical choice.

\subsection{Step 2: Optimize value of $\alpha$}

The previous step produces a number of different controllers corresponding to each value of $\alpha$ but it fails to provide an optimal value. This problem is nontrivial and a number of factors must be considered before choosing the optimal $\alpha$ value. It was found in 
simulations that decreasing the value of $\alpha$ produces an increment in the closed loop bandwidth (desirable for tracking high-frequency triangular trajectories). However, this comes at the cost of high settling time in its step response. Therefore, the value of $\alpha$ should be chosen by the design engineer based on the trajectory being tracked. Thus, the axis commanded to follow a triangular trajectory should utilize a small value of $\alpha$, and the axis commanded to follow a staircase reference should utilize an integer order controller.

However, in this paper we propose an optimization criterion based on the Integral of the Square Error (ISE) to take into account the reduction of performance in the time domain produced by small values of $\alpha$ in order to choose a definite value of $\alpha$. This criterion has been previously applied to the design of fractional order controllers. [25]. The ISE can be defined as:

$$
I S E=\int_{0}^{T}(r(t)-y(t))^{2} d t
$$

where $r(t)$ is the reference, $y(t)$ is the system output and $T$ is the duration of the experiment ( $T$ is set to $50 \mathrm{~ms}$ so that the response to the step input has enough time to reach the $\pm 3 \%$ error band for all values of $\alpha$ considered). The relationship between ISE and the different values of $\alpha$ obtained by simulation can be seen in Fig. 3. Additionally the relationship between the achievable bandwidth and the different values of $\alpha$ obtained by simulation is presented in Fig. 4

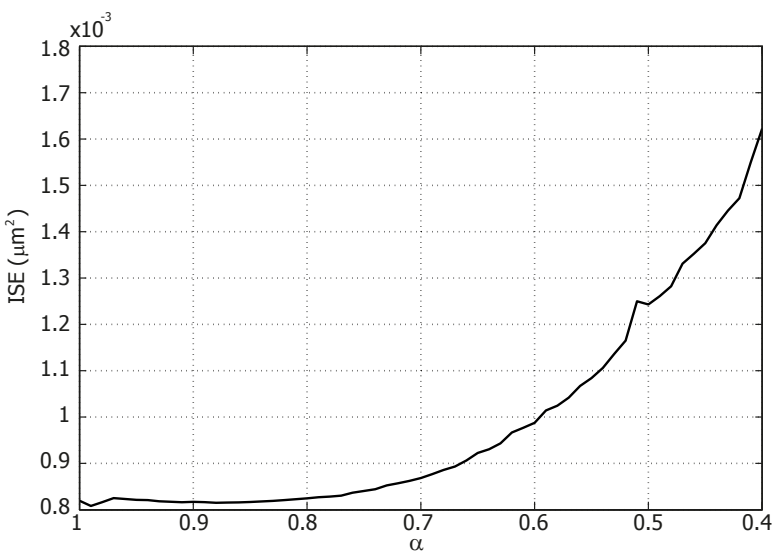

Figure 3: Evolution of the ISE for different values of $\alpha$

The aim of this work is to maximize a cost function which takes into account both frequency and the time domain response of the system. In order to define a cost function that exhibits a clear local maximum, a linear combination of an increasing function and a decreasing function is preferred. Figures 3 and 4 show that both the

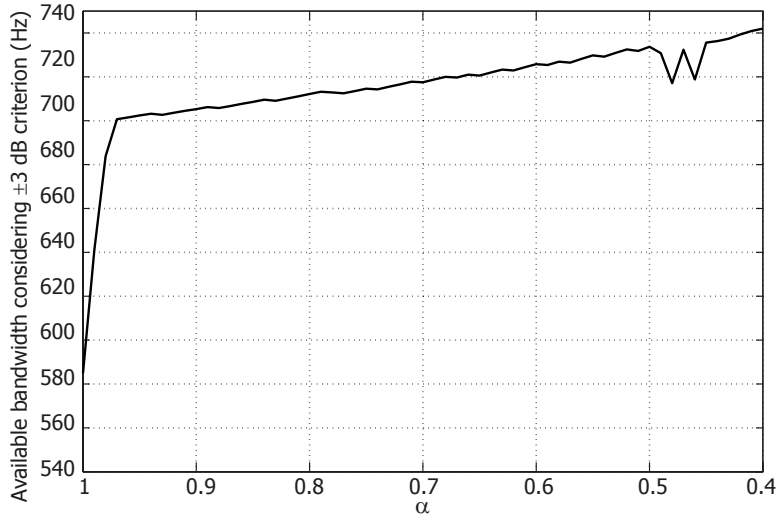

Figure 4: Evolution of the achievable bandwidth for different values of $\alpha$

ISE and the bandwidth functions are decreasing with $\alpha$. Then, instead of using the ISE index, its inverse - which is an increasing function with $\alpha$ - is used. Moreover, both functions, the bandwidth and the ISE inverse, are normalized in order to have the same range of variation between $[0,1]$. The two normalized functions that constitute the terms of the cost function are given below:

$$
\begin{gathered}
B W_{N o r m}=\frac{\omega_{b w}(\alpha)-\omega_{b w}(1)}{\omega_{b w}(0.4)-\omega_{b w}(1)} \\
\left(\frac{1}{I S E}\right)_{N o r m}=\frac{\frac{1}{I S E(\alpha)}-\frac{1}{I S E(0.4)}}{\frac{1}{I S E(1)}-\frac{1}{I S E(0.4)}}
\end{gathered}
$$

To optimize the performance of the designed control scheme, $\alpha$ is chosen so that the following cost function is maximized:

$$
J=\mu B W_{\text {Norm }}+(1-\mu)\left(\frac{1}{I S E}\right)_{\text {Norm }}
$$

where $\mu \in[0,1]$ is the weighting factor that defines the relative importance of the bandwidth and the timedomain response in the system's closed loop performance. For example, for a chosen value $\mu=0.5$, after computing the two successive optimization stages, the cost function $J$ presents the behaviour plotted in Fig. 5, and a global maximum of $\alpha=0.8$.

It is important to note that the results presented in Figs. 3 and 4 show that for values of $\alpha<0.5$, the ISE increases much faster than the bandwidth of the system and therefore, it was considered that values of $\alpha<0.4$ were conservatively considered to be outside the range of interest. 


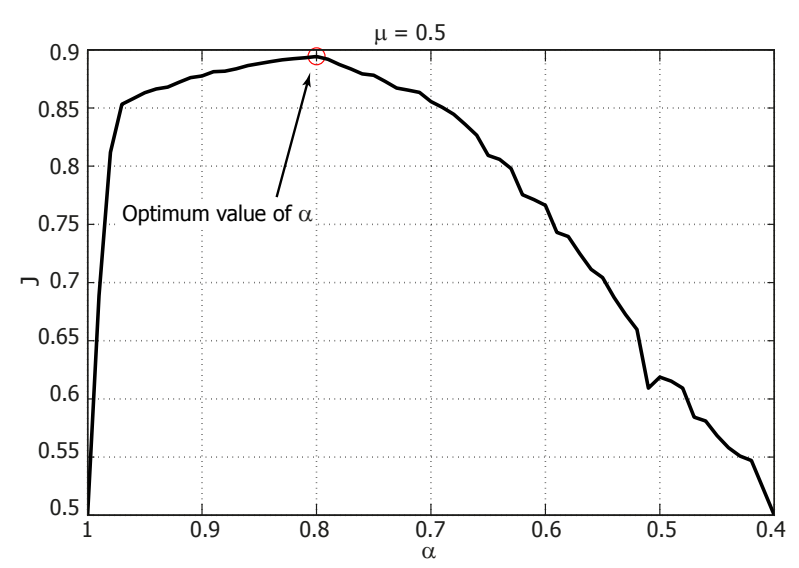

Figure 5: Evolution of the cost function for different values of $\alpha$

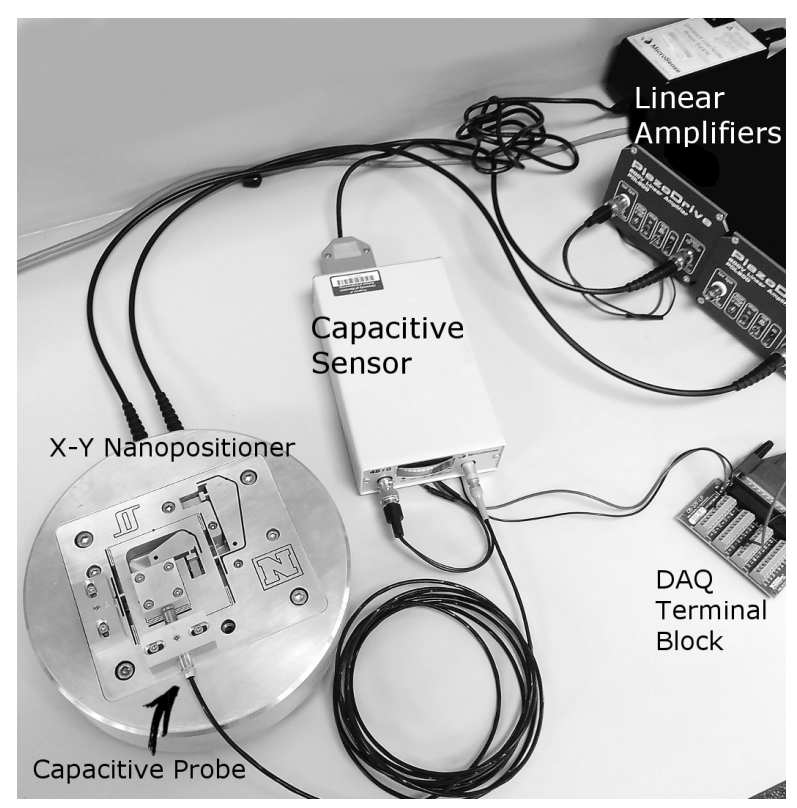

Figure 6: A two-axis serial kinematic nanopositioner, designed at the EasyLab, University of Nevada, Reno, driven by two PiezoDrive 200 $\mathrm{V}$ linear voltage amplifiers, with position measured by a Microsense 4810 capacitive sensor.

\section{Experimental setup}

In this section, the hardware utilized to test the performance of the proposed control scheme is described. This section also provides details on the identification procedure employed to characterize the experimental platform.

The experimental setup employed in this paper is shown in Fig. 6. The nanopositioner (designed at the EasyLab, University of Nevada, Reno, USA) is composed of a flexure XY serial mechanism driven by two Lead Zirconate Titanate (PZT) piezoelectric stack ac-
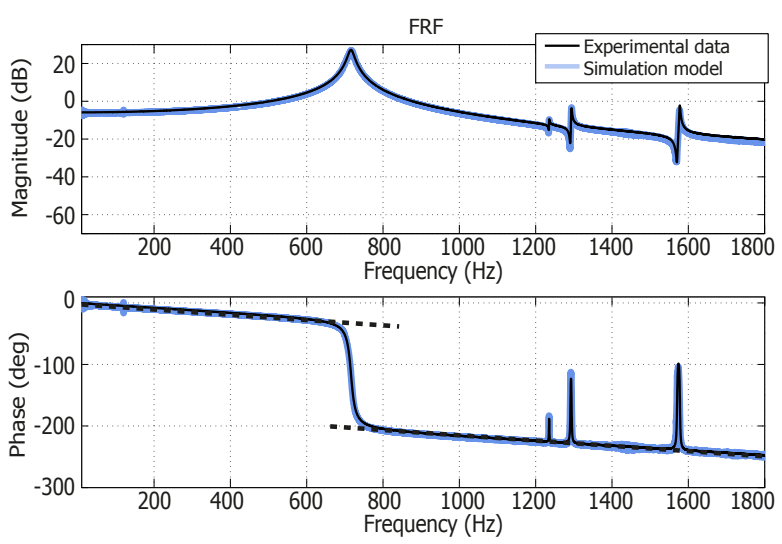

Figure 7: FRF of the $x$-axis of the experimental platform measured from the input voltage to output displacement.

tuators. The voltage applied to the PZT actuators is provided by two piezoelectric amplifiers which increase the voltage of the control signal by a gain factor of 20 and a bias of $100 \mathrm{~V}$. The nanopositioner delivers translational motion along each axis which is measured by a Microsense 4810 capacitive displacement sensor and a 2805 measurement probe with a sensitivity of $5 \mu \mathrm{m} / \mathrm{V}$. A PCI-6621 data acquisition card from National Instruments installed on a PC running the Real-Time Module from LabVIEW is used to interface between the experimental platform and the control design. The PC utilized is an OPTIPLEX 780 with an Intel(R) Core(TM)2 Duo Processor running at $3.167 \mathrm{GHz}$ and equipped with $2 \mathrm{~GB}$ of DDR3 RAM memory.

The cross-coupling between the two axes was measured to be $-40 \mathrm{~dB}$; small enough to be neglected, thereby making it feasible to treat each axis as being decoupled from the other. The $x$-axis of the platform is used to conduct the experiments presented in this work, while the $y$-axis was set to a bias of $100 \mathrm{~V}$ so as to mimic a realistic platform operation.

\subsection{System Identification}

A useable model of the nanopositioner was identified with sufficient accuracy through the use of small signal frequency response functions (FRFs). FRFs are determined by applying a small-amplitude sinusoidal $(0.2$ V) chirp signal (from 0.1 to $1800 \mathrm{~Hz}$ ) as input to the nanopositioner. Both the input and the output signals are then utilized to compute the FRFs by taking the Fourier transform of the recorded data. Fig. 7 shows the magnitude and phase responses of the FRF of $G(s)$ for a sampling time of $50 \mu \mathrm{s}$.

The chosen frequency range captures the first four resonance modes of the platform (at 716.2, 1235.5, 
1294, and $1578 \mathrm{~Hz}$ ) and also shows that the phase response appears to include a linear term (see the dotted line in Fig. 7), suggesting a time delay which supports the theoretical model (1).

The procedure utilized to obtain the transfer function of the system consists of two steps: first the resonance modes of the transfer function of the system were obtained by using the subspace based modelling technique described in [26], and then the delay was adjusted by minimizing the root-mean-square error (RMSE) of the phase response. The transfer function identified is

$$
\begin{aligned}
G_{4}(s, \tau) & =\frac{1.024 \times 10^{7} e^{-\tau s}}{s^{2}+99 s+2.025 \times 10^{7}}+\frac{10000 e^{-\tau s}}{s^{2}+7.76 s+6.026 \times 10^{7}} \\
& +\frac{62500 e^{-\tau s}}{s^{2}+13.01 s+6.61 \times 10^{7}}+\frac{122500 e^{-\tau s}}{s^{2}+15.86 s+9.83 \times 10^{7}}
\end{aligned}
$$

where: $\tau=115 \mu \mathrm{s}$.

The identified model accurately captures the dynamics of the platform axis within the bandwidth of interest $(\leq 1800 \mathrm{~Hz})$. It is important to note that despite it can seem that (8) use numeric values that exceed the maximum achievable precision by real-life deployment systems, this model is only utilized in the offline design of the parameters of the control scheme. The experimental implementation of the controller uses the numerical values of the gains showed in Table 1 (which only present up to 2 decimal places) and thus is easily implementable in practice. Additionally it was checked that by using IEEE 754 double-precision binary floating-point format numbers there were no numerical issues in the internal calculations of the algorithm and any discretization issue that might arise would be produced by the analog to digital conversion in the data acquisition card, but simulations showed that these errors were lower than $0.001 \%$.

\section{Simulations}

Simulations were performed using two different models derived from the measured frequency response in the previous section. A simple second order model considering only the first resonant mode was utilized throughout the design of the FIRC scheme. Once the optimization procedure was completed and the parameters of the FIRC were designed for the different values of $\alpha$, these controllers were simulated in closed loop scheme with the system model that included all the four resonant modes within the bandwidth of interest (8). This was done to verify the stability of the designed controllers even in the presence of high-frequency modes that were not considered in the design stage.

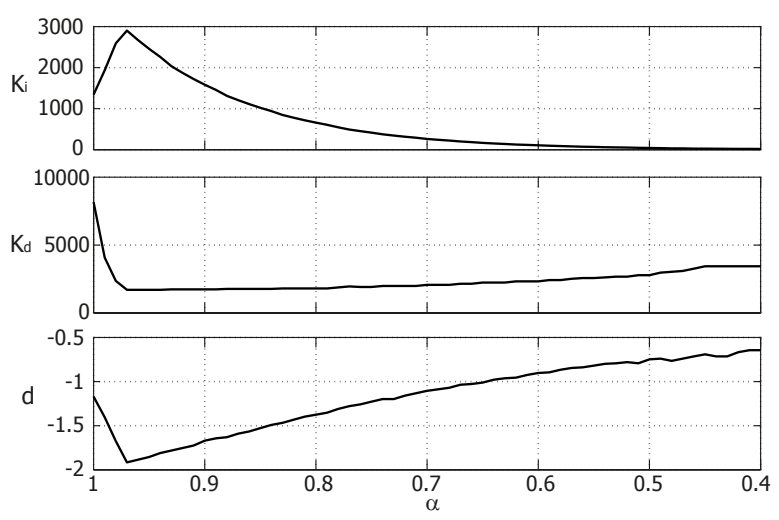

Figure 8: Evolutions of the parameters of the FIRC considering different values of $\alpha$.

\subsection{IRC design with delay compensation}

The mathematical model utilized to design the controller can be written as:

$$
H(s)=\frac{1.024 \times 10^{7} e^{-115 \times 10^{-6} s}}{s^{2}+99 s+2.025 \times 10^{7}}
$$

Using the equation presented in the appendix, the resulting IRC controller transfer function is:

$$
C_{t}(s)=\frac{950.3}{s}, C_{d}(s)=\frac{8852}{s}, d=-0.9757,
$$

It is important to note that these parameters are obtained by using the design methodology based on the Butterworth filter pattern presented in the appendix and these parameters are thus different from the ones computed by using the methodology based on the optimization procedure proposed in the following section.

\subsection{FIRC design with delay compensation}

Once the initial parameters values of the FIRC are obtained (the values of $K_{t c}, K_{d c}$ and $d$ of the traditional IRC with delay compensation), the optimization procedure described in Section 3.1 is applied to obtain the values of $K_{t c}, K_{d c}$ and $d$ that maximize the available bandwidth $\omega_{b w}$ of the closed loop system for different values of $\alpha$. The evolution of the parameters of the FIRC controller obtained during the design procedure ( $\Delta \alpha=0.01$ and $\alpha$ $\in[0.4,1])$ can be seen in Fig. 8, and parameters of the different controllers applied to the experimental system can be found in Table 1 .

From Fig. 8 it can be seen that there is a change in the behaviour of the evolution of the parameters of the FIRC that is produced when $\alpha=0.97$. This change corresponds to a change in the behaviour of the relationship between 
the bandwidth of the closed loop system and the value of $\alpha$. However, when these results were checked on the experimental platform, it was seen that changes in the value of $\alpha$ smaller than 0.1 produced indistinguishable results and that the change in the behaviour was produced closer to $\alpha=0.9$. This justifies using a single-point post-decimal resolution for $\alpha$ in Table 1 .

\begin{tabular}{|c|c|c|c|c|}
\hline$\alpha$ & $K_{t c}$ & $K_{d c}$ & $d$ & Bandwidth (Hz) \\
\hline 1 & 1343.0 & 8150.6 & -1.17 & 584.9 \\
\hline 0.9 & 1583.9 & 1735.9 & -1.67 & 705.3 \\
\hline 0.8 & 658.5 & 1806.0 & -1.38 & 712.2 \\
\hline 0.7 & 261.9 & 2070.5 & -1.11 & 717.5 \\
\hline 0.6 & 106.5 & 2329.0 & -0.90 & 725.8 \\
\hline 0.5 & 42.4 & 2780.2 & -0.75 & 733.7 \\
\hline 0.4 & 16.8 & 3435.7 & -0.64 & 742.0 \\
\hline
\end{tabular}

Table 1: Parameters of the FIRC considering the first decimal of $\alpha$.

In order to visualize the relationship between the closed loop bandwidth of the system and $\alpha$, the regulators derived from parameters of Table 1 were simulated and corresponding results are presented in Fig. 9. It is clear that the closed loop response of the system behaves like a low-pass filter with a very flat pass-band response (with a band pass ripple of $\delta_{\max }=1 \mathrm{~dB}$ ), and that using a fractional value of $\alpha$ produces a significant increment in the available bandwidth of the closed loop response. However, it is also important to note that as the fractional exponent takes lower values, the magnitude response of the closed loop system at lower frequencies decreases, which causes slower convergence to a steady state value. These two effects, the relationship between the value of the exponent and the bandwidth, and the slowly converging transient response, are expounded in more detail in the following section.

\subsection{Robustness analysis}

As stated earlier, the main advantage of the fractional order controller is its ability to increase the phase margin robustness of the closed loop system. The tracking scheme $C_{t}(s)$ of the FIRC can be written as:

$$
C_{C P E}(s)=C_{t}(s)=K_{t c} s^{\alpha},
$$

where $\alpha<0$. It is clear that this transfer function corresponds to the well-known constant phase element (CPE), [27]. This transfer function has a frequency response with a constant phase between the entire range of frequencies $0<\omega<\infty$, and allows regulator designs with desired phase margins. In order to show the robustness of the regulators parametrized in Table 1, the

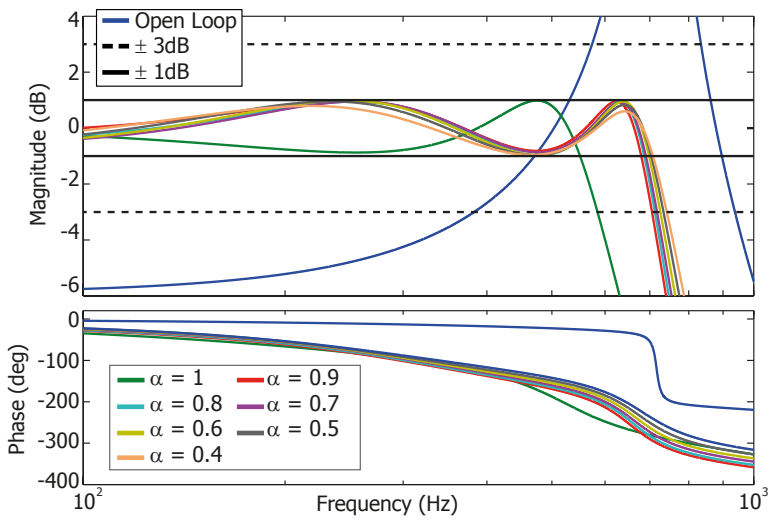

Figure 9: Open loop and closed loop frequency responses for FIRC with different values of $\alpha$ (Simulated results).

Nyquist plots of the different regulators were analyzed (considering the four vibration modes of the system) to obtain the phase margin $\Phi_{M}$, gain margin $M_{g}$, and the values of the frequencies at these points $\left(\omega_{c}\right.$ and $\left.\omega_{g}\right)$. These values are shown in Table 2 .

\begin{tabular}{|c|c|c|c|c|}
\hline$\alpha$ & $\Phi_{M}\left(^{\circ}\right)$ & $\omega_{c}(\mathrm{~Hz})$ & $M_{g}(\mathrm{~dB})$ & $\omega_{g}(\mathrm{~Hz})$ \\
\hline 1 & 65.0 & 171 & 5.57 & 489 \\
\hline 0.9 & 54.2 & 203 & 6.25 & 532 \\
\hline 0.8 & 54.6 & 209 & 6.27 & 545 \\
\hline 0.7 & 54.6 & 216 & 6.12 & 559 \\
\hline 0.6 & 53.3 & 221 & 6.03 & 574 \\
\hline 0.5 & 53.3 & 225 & 5.91 & 590 \\
\hline 0.4 & 53.7 & 227 & 5.86 & 607 \\
\hline
\end{tabular}

Table 2: Representative points of the Nyquist diagram for the different controllers designed depending on $\alpha$.

From the values given in Table 2, it is clear that phase margin and gain margin remain more or less unchanged for all the designed controllers. This implies that these parameters, usually associated with stability robustness and damping robustness, are barely affected by changes in $\alpha$. Moreover, these results show that the phase margin is also guaranteed for all resonant modes, thereby avoiding the detrimental effects due to unmodelled spillover dynamics [28]. Compared to the integer order controller, the phase margin decreases while the gain margin increases.

\section{Experimental Results}

The performance of the proposed control scheme was experimentally validated in the two-axis serial kinematic nanopositioner described in Section 4. These experimental results show the change in the behaviour of 
the closed loop system as the value of $\alpha$ is decreased and highlight that the effectiveness of a fractional order controller is determined by the type of reference needed in each application.

The fractional exponent of the proposed controller is implemented in the experimental platform by using the following expression:

$$
C_{t}(s)=\frac{K_{t c}}{s^{\alpha}}=\frac{K_{t c}}{s} s^{\gamma}
$$

where $\gamma=1-\alpha$. The fractional order differential operator of this expression is discretized by using the Grünwald-Letnikov (GL) definition of the discretized fractional operator [29], and the short memory approximation [30], as follows:

$$
y_{c}(t)=T_{s}^{\gamma} \sum_{j=0}^{N-1}(-1)^{j}\left(\begin{array}{l}
\gamma \\
j
\end{array}\right) f\left(t-j T_{s}\right) ;
$$

where the integral of the error signal $(r-y)$ multiplied by $K_{t c}, f(t)$, is the input to the block $s^{\gamma}, y_{c}(t)$ is its output, $N=200$ is the number of terms involved in the discrete convolution, $T_{s}$ is the sampling time, and the combinatorial has been generalised in the following respect:

$$
\left(\begin{array}{l}
\gamma \\
j
\end{array}\right)=\frac{\gamma(\gamma+1) \ldots(\gamma-j+1)}{j !} .
$$

\subsection{Frequency-domain results}

The well-known criterion of $\pm 3 \mathrm{~dB}$ bandwidth is widely employed in order to quantify the performance of different control schemes as regards nanopositioning systems (because it provides a measurement of the bandwidth of the signals which can be tracked without significant distortion). In this work, the controllers were designed using a $\pm 1 \mathrm{~dB}$ restriction, and the criterion of $\pm 3 \mathrm{~dB}$ was utilized to determine the experimental bandwidth achieved by each controller. This approach effectively eliminated any issues introduced by uncertainties in the identified model of the nanopositioner. The closed loop frequency responses obtained experimentally are presented in Fig. 10 and the $\pm 3 \mathrm{~dB}$ bandwidths achieved via simulations and via experiments are tabulated in Table 3. Experimental and simulated results are in good agreement in all the cases.

The results shown in Fig. 10 demonstrate that the closed loop bandwidth can be increased by decreasing the value of $\alpha$. Furthermore, as stated in Section 5, there is a change in the relationship trend between the bandwidth and the value of $\alpha$. In the experimental system, this point was at $\alpha=0.9$. This evolution can be seen in Fig. 11.

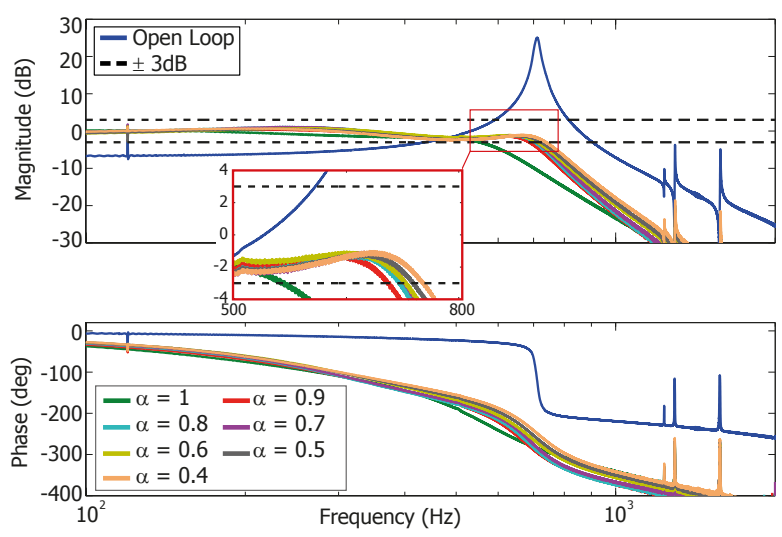

Figure 10: Open loop and closed loop frequency response for FIRC with different values of $\alpha$. Experimental results

\begin{tabular}{|c|c|c|}
\hline$\alpha$ & $\begin{array}{c}\text { Experimental } \\
\text { Bandwidth }(\mathrm{Hz})\end{array}$ & $\begin{array}{c}\text { Simulated } \\
\text { Bandwidth }(\mathrm{Hz})\end{array}$ \\
\hline 1 & 549.8 & 584.9 \\
\hline 0.9 & 685.2 & 705.3 \\
\hline 0.8 & 702.4 & 712.2 \\
\hline 0.7 & 709.2 & 717.5 \\
\hline 0.6 & 710 & 725.8 \\
\hline 0.5 & 722.6 & 733.7 \\
\hline 0.4 & 736.4 & 742.0 \\
\hline
\end{tabular}

Table 3: Achievable bandwidth for different values of $\alpha$.

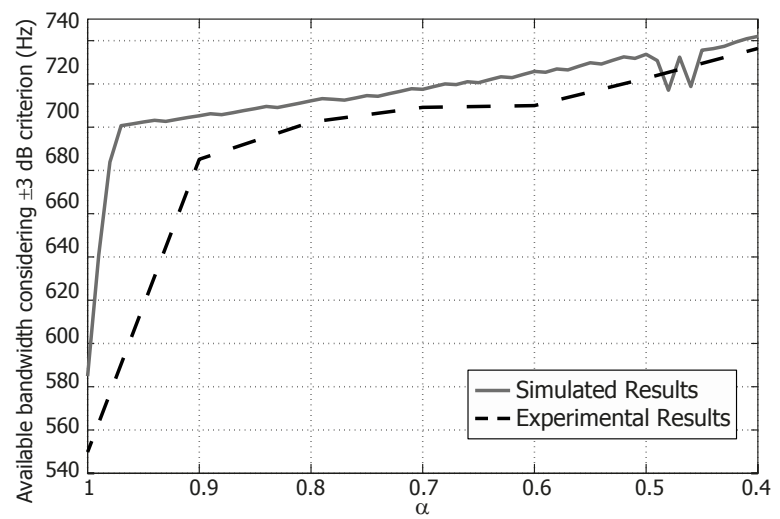

Figure 11: Relationship between achievable bandwidth and $\alpha$.

\subsection{Time-domain results}

In order to quantify the performance of the FIRC scheme in the time-domain, two commonly used reference signals typically employed in nanopositioning were utilised, namely triangular and step signals. 


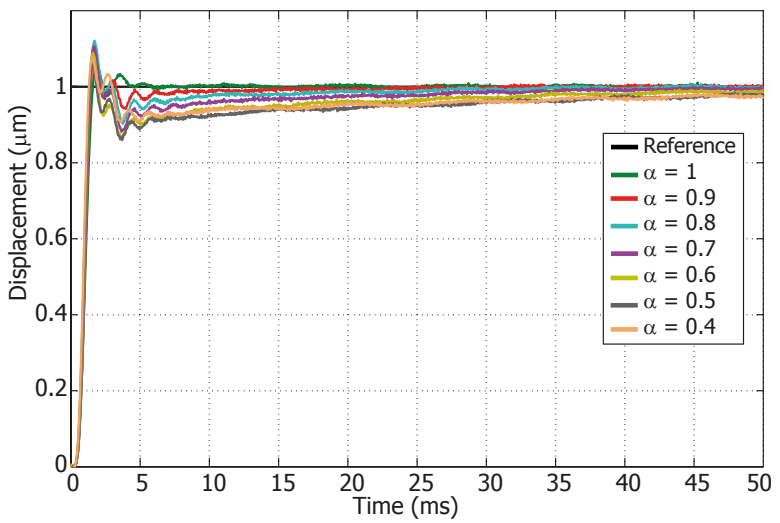

Figure 12: Measured closed loop response of the FIRC to a step signal with different values of $\alpha$.

\subsubsection{Step reference}

As discussed earlier, $\alpha=0.4$ was the lowest value used in this work. The recorded closed loop responses of the system to a unity step signal for different values of $\alpha$ are plotted in Fig. 12.

It can be seen from Fig. 12 that the steady state tracking error is zero because of the integral action provided by the FIRC. However, it can also be seen that the lower the value of $\alpha$, the slower the convergence to steady state. This deterioration of the performance in the time domain with the reduction of $\alpha$ can be mitigated in two ways:

- By setting a limit to the lower value that can be assigned to $\alpha$ as in (7), where there is a penalty associated to slow transient responses.

- By adding a pre-filter $F(s)$ to the control scheme as proposed in [31].

Simulation results show that a pre-filter of the form:

$$
F(s)=\frac{s^{\alpha}\left(d \omega^{2}-\sigma^{2}\right)+K_{t c} \sigma^{2}}{K_{t c} \sigma^{2}(1+\lambda s)}
$$

where $\lambda$ delivers substantial improvement in the transient response of the closed loop system, see Fig. 13. However, using a pre-filter may decrease the closed loop bandwidth of the transfer function between $r(t)$ and $y(t)$.

\subsubsection{Triangular references}

In order to quantify the triangular trajectory tracking performance of the proposed scheme, triangular waves with frequencies in the interval $[0,200] \mathrm{Hz}$ and amplitude of $1 \mu \mathrm{m}$ were applied to the closed loop system for the different values of $\alpha$. The results obtained enable the determination of the relationship between Root

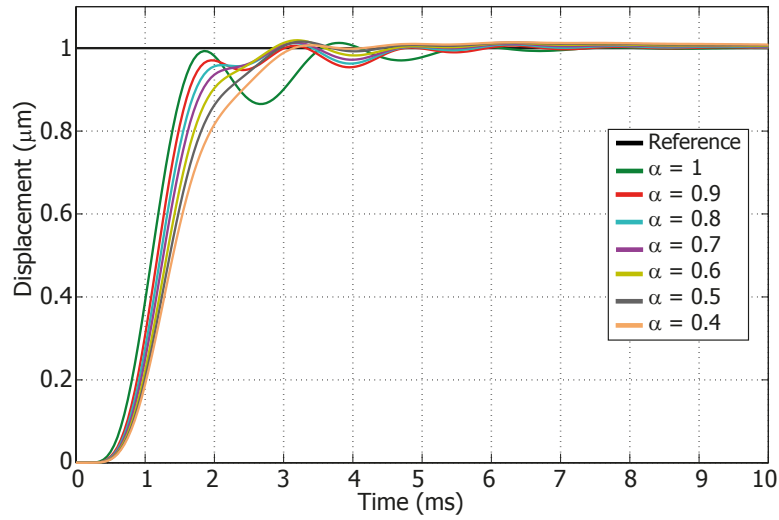

Figure 13: Simulated closed loop response of the FIRC to a step signal with different values of $\alpha$ and a prefilter applied to the controller.

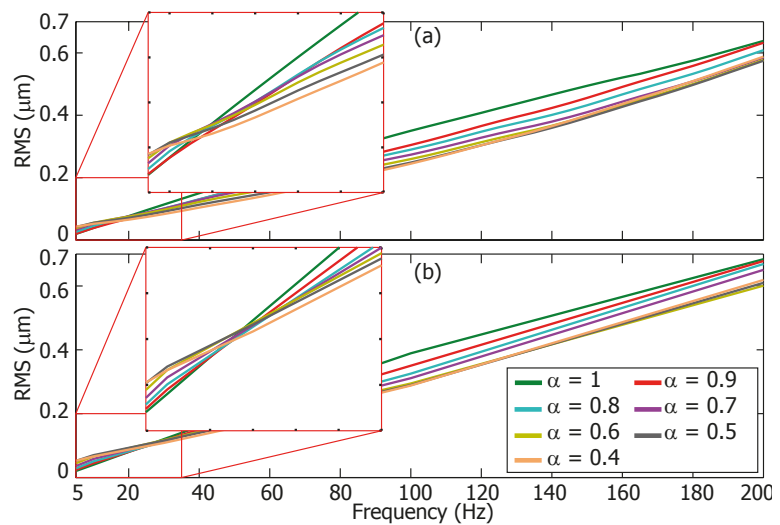

Figure 14: Root-mean-square error in the tracking of triangular signals of different frequencies depending on the value of $\alpha$ (a) Simulated results, and (b) Experimental results.

Mean Square Error (RSME) while tracking triangular trajectories of different frequencies and the value of $\alpha$. These relationships are plotted in Fig. 14 for both the simulated and the experimental results.

From Fig. 14, it can be seen that the experimental and simulated results are in good agreement, and that, in all the cases, there is an almost linear relationship between the RMSE and the frequency of the triangular signal (a foreseeable result, knowing that there is a limited closed loop bandwidth in all the cases). Additionally, it can be seen that while tracking triangular trajectories at higher frequencies, the RMSE decreases with decrease in the value of $\alpha$ due to increased closed loop bandwidth. However, the result is the opposite for lower frequencies due to higher settling times. This remarkable behaviour has to be accounted for in the design of the FIRC, i.e. if the controller is designed to track slow triangular signals (with a frequency lower than 30 
$\mathrm{Hz}$ in our experimental system) it is desirable to choose high values of $\alpha$. However, if we want to track high frequency signals, lower values of $\alpha$ will produce results with less RMSE.

Finally, in order to assess the effects of the technique proposed in the Appendix A in compensating the effects of the delay in the system, the traditional IRC scheme without delay compensation as in [13], and the traditional IRC scheme with delay compensation were experimentally compared. The bandwidth achieved was $212 \mathrm{~Hz}$ for the traditional IRC without delay compensation, and $354 \mathrm{~Hz}$ for the traditional IRC scheme with delay compensation. It is important to note that the delay compensation alone increases the closed loop bandwidth by $67 \%$. Additionally, when the FIRC proposed in this paper is implemented, the available bandwidth can be further increased to $247 \%$ with respect the traditional IRC scheme using $\alpha=0.4$ is utilized (computed as $((736.4-212) / 212) \cdot 100)$.

\section{Conclusions}

This paper proposes the design of a combined damping and tracking scheme where the classical Integral Resonant Control (IRC) is combined with a fractional order integral controller to deliver tracking. The fractional order integral controller introduces a new design parameter that facilitates increasing the achievable closed loop positioning bandwidth while keeping a flat pass-band response. Both simulations and experiments present good agreement and demonstrate an improvement in achievable bandwidth by $247 \%$.

It is further demonstrated that selection of the fractional order depends on the trajectory to be tracked. It is shown that in the case of step commands, the apt order may be obtained via an optimization procedure that establishes a compromise between the closed loop bandwidth and the ISE index. However, in case of triangular trajectories, fractional order controllers produce superior performance because of the higher bandwidth that can be obtained, while for low frequency signals (below $20 \mathrm{~Hz}$ ), the integer order controller yields the best performance due to low output errors.

\section{Appendix A. Exact design of IRC in presence of de- lay in the system}

In the case of $\alpha=1$ the characteristic equation of (3) becomes:

$$
\sigma^{2} K_{d c}\left(K_{t c}-s\right)+\left(s^{3}+s^{2} 2 \zeta \omega+s \omega^{2}\right) e^{s \tau}\left(s-d K_{d c}\right)=0
$$

In order to devise a controller tuning method with which to place the poles, the characteristic equation is expressed as:

$$
\begin{array}{r}
-K_{d c} \sigma^{2} s+K_{d c} K_{t c} \sigma^{2} \\
-d K_{d c}\left(s^{3}+s^{2} 2 \zeta \omega+s \omega^{2}\right) e^{s \tau}= \\
-\left(s^{4}+s^{3} 2 \zeta \omega+s^{2} \omega^{2}\right) e^{s \tau}
\end{array}
$$

It can be seen that in this case there are only three parameters to design $K_{d c}, K_{t c}$ and $d$, and four poles of the closed loop system $z_{1}, z_{2}, z_{3}$ and $z_{4}$. Since we are interested on placing the closed loop poles of the system in a Butterworth filter pattern, the poles will present the following expressions:

$z_{1}=\rho e^{j \theta_{1}}, z_{2}=\rho e^{j \theta_{2}}, z_{3}=\rho e^{-j \theta_{1}}$ and $z_{4}=\rho e^{-j \theta_{2}}$, where $\rho$ is the radius of the circle along the closed loop poles are distributed, and $\theta_{1}$ and $\theta_{2}$ are the angles which determine the location of the poles $\left(\theta_{1}=7 / 8 \cdot \pi \mathrm{rad}\right.$, $\theta_{2}=5 / 8 \cdot \pi$ rad for a fourth order Butterwoth filter). The conditions that allow to tune the parameters become:

$$
\begin{array}{r}
-K_{d c} \sigma^{2} z_{i}+K_{d c} K_{t c} \sigma^{2} \\
-d K_{d c}\left(z_{i}^{3}+z_{i}^{2} 2 \zeta \omega+z_{i} \omega^{2}\right) e^{z_{i} \tau}= \\
-\left(z_{i}^{4}+z_{i}^{3} 2 \zeta \omega+z_{i}^{2} \omega^{2}\right) e^{z_{i} \tau}
\end{array}
$$

Only conditions $i=1,2$ are needed since the others are their complex conjugate counterparts. If we denote:

$$
\begin{gathered}
\Psi_{i}=-\left(z_{i}^{3}+z_{i}^{2} 2 \zeta \omega+z_{i} \omega^{2}\right) e^{z_{i} \tau} \\
\Xi_{i}=-\left(z_{i}^{4}+z_{i}^{3} 2 \zeta \omega+z_{i}^{2} \omega^{2}\right) e^{z_{i} \tau}
\end{gathered}
$$

yields the following system of equations:

$$
\left[\begin{array}{ccc}
-\sigma^{2} \rho \cos \left(\theta_{1}\right) & \sigma^{2} & \mathfrak{R}\left(\Psi_{1}\right) \\
-\sigma^{2} \rho \sin \left(\theta_{1}\right) & 0 & \mathfrak{J}\left(\Psi_{1}\right) \\
-\sigma^{2} \rho \cos \left(\theta_{2}\right) & \sigma^{2} & \mathfrak{R}\left(\Psi_{2}\right) \\
-\sigma^{2} \rho \sin \left(\theta_{2}\right) & 0 & \mathfrak{J}\left(\Psi_{2}\right)
\end{array}\right] \cdot\left[\begin{array}{c}
K_{d c} \\
K_{d c} K_{t c} \\
K_{d c} d
\end{array}\right]=\left[\begin{array}{c}
\mathfrak{R}\left(\Xi_{1}\right) \\
\mathfrak{J}\left(\Xi_{1}\right) \\
\mathfrak{R}\left(\Xi_{2}\right) \\
\mathfrak{J}\left(\Xi_{2}\right)
\end{array}\right]
$$

which has a solution if:

$$
\left|\begin{array}{cccc}
-\sigma^{2} \rho \cos \left(\theta_{1}\right) & \sigma^{2} & \mathfrak{R}\left(\Psi_{1}\right) & \mathfrak{R}\left(\Xi_{1}\right) \\
-\sigma^{2} \rho \sin \left(\theta_{1}\right) & 0 & \mathfrak{J}\left(\Psi_{1}\right) & \mathfrak{J}\left(\Xi_{1}\right) \\
-\sigma^{2} \rho \cos \left(\theta_{2}\right) & \sigma^{2} & \mathfrak{R}\left(\Psi_{2}\right) & \mathfrak{R}\left(\Xi_{2}\right) \\
-\sigma^{2} \rho \sin \left(\theta_{2}\right) & 0 & \mathfrak{J}\left(\Psi_{2}\right) & \mathfrak{J}\left(\Xi_{2}\right)
\end{array}\right|=0
$$

and all the columns of (A.6) are independent.

Obtaining the parameters of the IRC in the presence of delay involves therefore the following steps: 
1. Solve (A.6) to obtain the value of $\rho$ for a known value of $\tau$.

2. Introduce the value of $\rho$ and $\tau$ in (A.5) to obtain the values of $K_{t c}, K_{d c}$, and $d$

\section{References}

[1] I. A. Mahmood, S. O. R. Moheimani, Fast spiral-scan atomic force microscopy, Nanotechnology 20 (36) (2009) 365503.

[2] S. Devasia, E. Eleftheriou, S. O. R. Moheimani, A survey of control issues in nanopositioning, IEEE Transactions on Control Systems Technology 15 (4) (2007) 689 - 703.

[3] Y. K. Yong, S. S. Aphale, S. O. R. Moheimani, Design, identification, and control of a flexure-based xy stage for fast nanoscale positioning, IEEE / ASME Transactions on Mechatronics 8 (1) (2009) $46-54$

[4] J. L. Fanson, T. K. Caughey, Positive position feedback control for large space structures, AIAA Journal 28 (4) (1990) 717 724.

[5] B. Bhikkaji, M. Ratnam, A. J. Fleming, S. O. R. Moheimani, High-performance control of piezoelectric tube scanners, IEEE Transactions on Control Systems Technology 15 (5) (2007) 853 $-866$.

[6] S. S. Aphale, A. J. Fleming, S. O. R. Moheimani, Integral resonant control of collocated smart structures, Smart Materials and Structures 16 (2007) $439-446$.

[7] A. Preumont, B. de Marneffe, A. Deraemaeker, F. Bossens, The damping of a truss structure with a piezoelectric transducer, Computers and Structures 86 (2008) $227-239$.

[8] A. J. Fleming, S. S. Aphale, S. O. R. Moheimani, A new method for robust damping and tracking control of scanning probe microscope positioning stages, IEEE Transactions on Nanotechnology 9 (4) (2010) 438-448. doi:10.1109/TNANO.2009.2032418.

[9] B. Bhikkaji, S. O. R. Moheimani, Integral resonant control of a piezoelectric tube actuator for fast nanoscale positioning, IEEE/ASME Transactions on Mechatronics 13 (5) (2008) 530537. doi:10.1109/TMECH.2008.2001186.

[10] M. Namavar, A. J. Fleming, M. Aleyaasin, K. Nakkeeran, S. S. Aphale, An analytical approach to integral resonant control of second-order systems, IEEE/ASME Transactions on Mechatronics 19 (2) (2014) 651-659. doi:10.1109/TMECH.2013.2253115.

[11] D. Russell, A. J. Fleming, S. S. Aphale, Simultaneous optimization of damping and tracking controller parameters via selective pole placement for enhanced positioning bandwidth of nanopositioners, Journal of Dynamic Systems Measurement and Control 137 (10) (2015) 2184-2189. doi:10.1115/1.4030723.

[12] A. A. Eielsen, M. Vagia, J. T. Gravdahl, K. Y. Pettersen, Damping and tracking control schemes for nanopositioning, IEEE/ASME Transactions on Mechatronics 19 (2) (2014) 432444. doi:10.1109/TMECH.2013.2242482.

[13] D. Russell, A. San-Millan, V. Feliu, S. S. Aphale, Butterworth pattern based simultaneous damping and tracking controller designs for nanopositioning systems, Frontiers in Mechanical Engineering 2 (2). doi:10.3389/fmech.2016.00002.

[14] A. San-Millan, D. Russell, V. Feliu, S. S. Aphale, A modified positive velocity and position feedback scheme with delay compensation for improved nanopositioning performance, Smart Materials and Structures 24 (7) (2015) 075021.

[15] C. A. Monje, B. M. Vinagre, V. Feliu, Y. Chen, Tuning and auto-tuning of fractional order controllers for industry applications, Control Engineering Practice 16 (7) (2008) 798 - 812 doi:http://dx.doi.org/10.1016/j.conengprac.2007.08.006.
[16] M. Salehtavazoei, M. Tavakoli-Kakhki, Compensation by fractional-order phase-lead/lag compensators, IET Control Theory Applications 8 (5) (2014) 319-329. doi:10.1049/ietcta.2013.0138

[17] C. A. Monje, F. Ramos, V. Feliu, B. M. Vinagre, Tip position control of a lightweight flexible manipulator using a fractional order controller, IET Control Theory Applications 1 (5) (2007) 1451-1460. doi:10.1049/iet-cta:20060477.

[18] G. W. Bohannan, Analog fractional order controller in temperature and motor control applications, Journal of Vibration and Control 14 (9-10) (2008) 1487-1498. doi: $10.1177 / 1077546307087435$

[19] D. Feliu-Talegon, A. San-Millan, V. Feliu-Battle, Fractionalorder integral resonant control of collocated smart structures, Control Engineering Practice 56 (2016) 210 - 223. doi:http://dx.doi.org/10.1016/j.conengprac.2016.07.016

[20] S. S. Aphale, A. J. Fleming, S. O. R. Moheimani, Integral resonant control of collocated smart structures, Smart Materials and Structures 16 (2) (2007) 439-446.

[21] S. S. Aphale, B. Bhikkaji, S. O. R. Moheimani, Minimizing scanning errors in piezoelectric stack-actuated nanopositioning platforms, IEEE Transactions on Nanotechnology 7 (1) (2008) 79-90. doi:10.1109/TNANO.2007.910333.

[22] A. Acharya, S. Das, I. Pan, S. Das, Extending the concept of analog Butterworth filter for fractional order systems, Signal Processing 94 (2014) 409 - 420. doi:http://dx.doi.org/10.1016/j.sigpro.2013.07.012

[23] C. Matos, M. D. Ortigueira, Fractional Filters: An Optimization Approach, Springer Berlin Heidelberg, Berlin, Heidelberg, 2010.

[24] T. Freeborn, B. Maundy, A. S. Elwakil, Approximated fractional order Chebyshev lowpass filters, Mathematical Problems in Engineering 2015 (2015) 7. doi:http://dx.doi.org/10.1155/2015/832468.

[25] Y. Luo, Y. Chen, Fractional Order Motion Controls, Wiley, 2012.

[26] T. McKelvey, H. Akcay, L. Ljung, Subspace-based multivariable system identification from frequency response data, IEEE Transactions on Automatic Control 41 (7) (1996) 960-979. doi: $10.1109 / 9.508900$.

[27] K. S. Cole, Electric conductance of biological systems, in: Cold Spring Harbor symposia on quantitative biology, Vol. 1, Cold Spring Harbor Laboratory Press, 1933, pp. 107-116.

[28] R. L. Clark, Accounting for out-of-bandwidth modes in the assumed modes approach: implications on collocated output feedback control, Trans. ASME Journal of Dynamic Systems, Measurement, and Control 119 (1997) 390 - 395.

[29] B. M. Vinagre, I. Podlubny, A. Hernandez, V. Feliu, Some approximations of fractional order operators used in control theory and applications, Fractional Calculus and Applied Analysis 3 (3) (2000) 231-248.

[30] I. Podlubny, Fractional differential equations: an introduction to fractional derivatives, fractional differential equations, to methods of their solution and some of their applications, Vol. 198, Academic Press, 1998.

[31] V. Feliu-Batlle, R. Rivas-Perez, Robust fractionalorder controller for an EAF electrode position system, Control Engineering Practice 56 (2016) 159 - 173. doi:http://dx.doi.org/10.1016/j.conengprac.2016.04.017. 
LaTeX Source Files
Click here to download LaTeX Source Files: Fractional_Nano.zip

LaTeX Source Files
Click here to download LaTeX Source Files: Fractional_Nano.zip Click here to download LaTeX Source Files: Fractional_Nano.zip

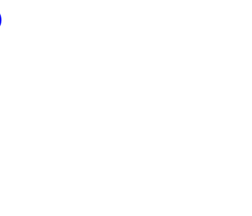
(1) (1) (1)

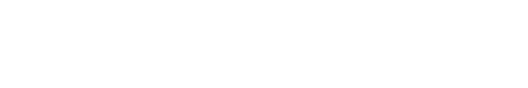
- n . .

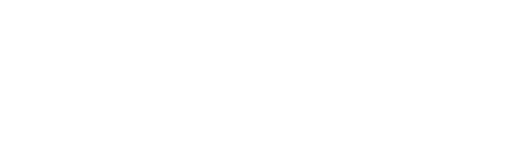

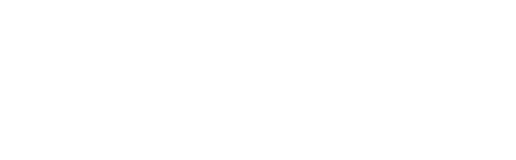
.

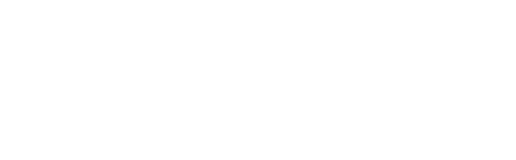

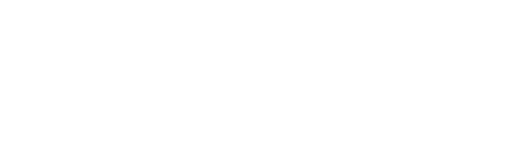

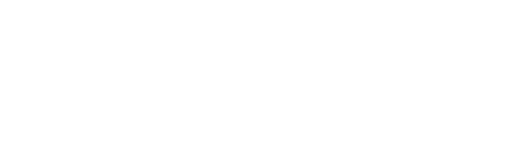

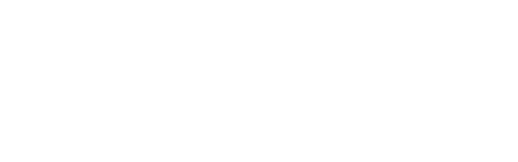

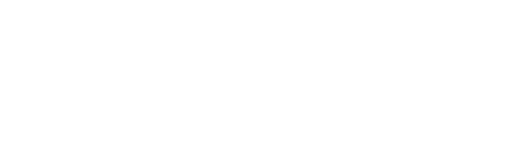

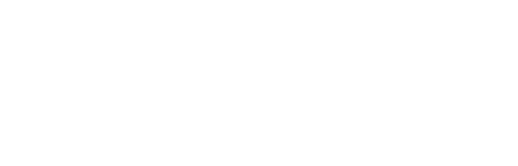

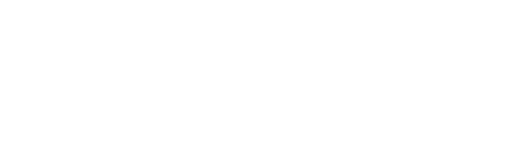

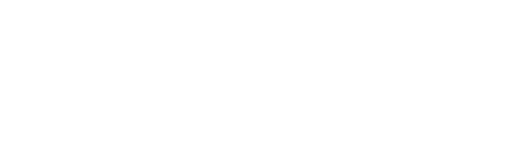

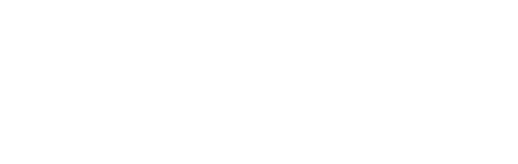

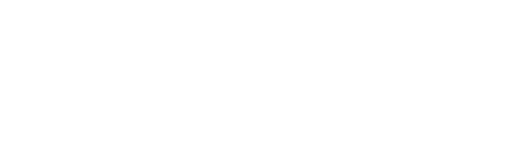

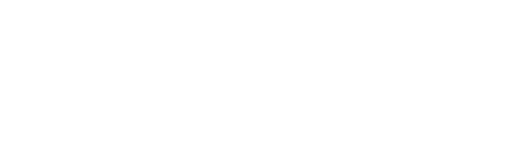

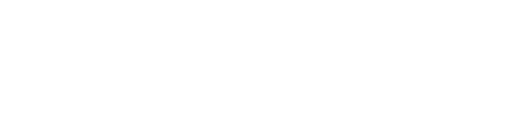

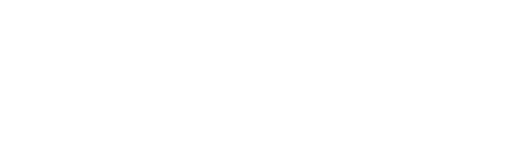

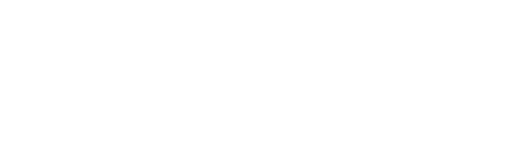

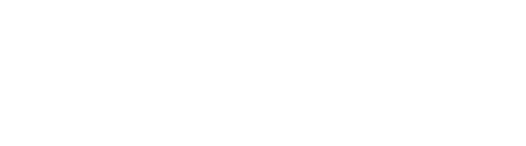

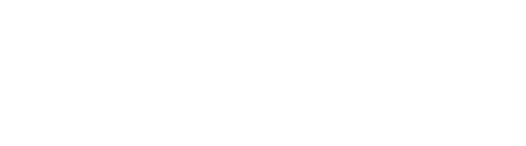

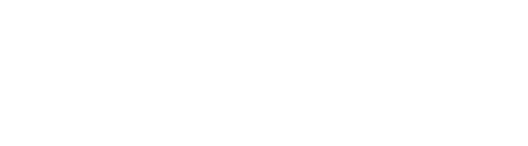

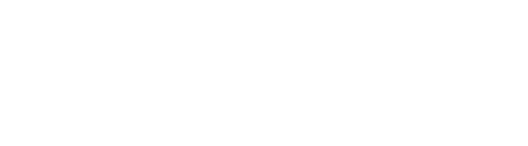
. . . 